\title{
All Cervical Sonography Is Not Created Equal
}

\author{
Cord Sturgeon, MD, MS, FACS
}

Department of Surgery, Division of Endocrine Surgery, Northwestern Memorial Hospital, Northwestern University Feinberg School of Medicine, Chicago, Illinois

Because papillary thyroid cancer (PTC) is frequently associated with cervical nodal metastases, and because the detection and removal of these metastatic nodes is believed to translate to a lower rate of recurrent or persistent disease, it is desirable to detect these nodal metastases preoperatively and properly address them during the index thyroid operation. It stands to reason that the most sensitive and accurate preoperative nodal staging should be associated with the greatest likelihood of applying the most expedient surgical procedure while minimizing unnecessary dissection. In this issue of Annals of Surgical Oncology, Oltmann and colleagues report on the recurrence rate in patients who underwent what they believe to be the most sensitive method of preoperative nodal staging, surgeon-performed ultrasound. They compare recurrence rates between patients who had preoperative cervical ultrasound performed by surgeons and nonsurgeons. Although this is a small retrospective study, the findings raise the question, "Are all cervical sonograms created equal?"

Cervical nodal metastases are found in $30-80 \%$ of cases of PTC, are most commonly located in the central compartment (level VI), and are a risk factor for disease persistence or recurrence. ${ }^{1-3}$ Preoperative physical exam has a very low sensitivity and specificity for the detection of cervical nodal metastases. ${ }^{4,5}$ Furthermore, surgeons are notoriously poor at even the intraoperative detection of nodal metastases. ${ }^{6}$ Preoperative ultrasound is now the standard modality for evaluating cervical nodal status in patients with thyroid cancer because it is much more sensitive and specific than physical exam. A meta-analysis found that cervical ultrasound is $70 \%$ sensitive and $98 \%$

(C) Society of Surgical Oncology 2014

First Received: 31 August 2014;

Published Online: 19 September 2014

C. Sturgeon, MD, MS, FACS

e-mail: csturgeo@nmh.org specific for the preoperative detection of nodal metastases. ${ }^{7}$ Sonographic clues that herald the presence of metastatic PTC within a cervical node are well-described and can be found by examining nodal morphology, vascularity, size, and echogenicity. ${ }^{8-11}$

Several studies have shown that a preoperative cervical ultrasound frequently leads to the detection of occult nodal disease that changes the extent of surgery. ${ }^{4,5,12-14}$ Nonpalpable nodal metastases may be detected in roughly 20 $40 \%$ of patients with PTC and will alter the operative approach in a similar percentage of patients. The findings from these studies emphasize the importance of a comprehensive, high-quality, high-resolution, preoperative cervical ultrasound for thyroid cancer patients. Knowledge of the presence and location of nodal metastases before the index operation for thyroid cancer improves the chance that an appropriate lymph node clearance will be planned and performed. Accordingly, in guideline statements, the American Thyroid Association (ATA) and the National Comprehensive Cancer Network (NCCN) both recommend preoperative cervical ultrasound for all patients with papillary thyroid cancer. ${ }^{15,16}$

There is considerable controversy regarding the management of the clinically NO central nodal basin during an index thyroid operation, but there is no controversy regarding the management of clinically or pathologically N1 central nodal basins. ${ }^{17}$ A preoperative knowledge of metastatic disease allows the surgeon to have a more accurate discussion with the patient about the risks of surgery and the probability of recurrence. Furthermore, it allows the surgeon to plan a formal nodal clearance and to allot the appropriate amount of time and resources to the operation.

Surgeon-performed ultrasound has been found to be highly effective in the detection and prognostication of thyroid nodules and cervical lymph node metastases. ${ }^{12,13,18,19}$ The findings from some studies suggest that surgeon-performed ultrasound, may even be superior to 
ultrasound performed in radiology departments in some institutions. ${ }^{12,20}$ Oltmann and colleagues expand on this concept and take their hypothesis one step further. The hypothesis of the current study was that surgeon-performed ultrasound is not only superior to ultrasound performed in a radiology department but also its usage may be associated with a lower rate of cancer recurrence.

Oltmann and colleagues found that the recurrence rate was $0 \%$ over a mean follow-up of 20 months in the group who underwent surgeon-performed ultrasound. In comparison, the recurrence rate was $12 \%$ over 34 months in the group of patients who received an ultrasound in the radiology department. The authors concluded that a negative high-quality preoperative ultrasound may obviate the need for central neck dissection.

When critically reviewing this study, the reader should bear in mind that there are several limitations that might affect the findings. First, this is actually a comparison of the outcomes of a single surgeon with dedicated ultrasound training who performs his or her own cervical ultrasounds, versus those of other surgeons who obtained ultrasound examinations in a radiology department. There are no dedicated thyroid sonographers at the authors' institution, and therefore, there may be a difference in sensitivity between the surgeon-performed ultrasound and the one performed in the radiology department that might simply be due to a volume-outcome relationship. This is not a prospective randomized study, and therefore, it suffers from biases attributable to retrospective reviews. The cohort of patients who underwent surgeon-performed ultrasound made up only approximately $27 \%$ of the entire group. Ninety-four percent of patients in the surgeon-performed ultrasound group received radioiodine ablation (RAI) with a mean dose of $93 \mathrm{mCi}$, whereas $88 \%$ of the nonsurgeon ultrasound group received RAI with a mean dose of $83 \mathrm{mCi}$. These proportions were not significantly different. Follow-up was significantly shorter in the surgeon-performed ultrasound group (20 vs. 34 months). Disease recurrence was defined as need for a second dose of RAI, a positive fine needle aspiration biopsy, or requiring a second operation where cancer was identified on final pathology. Recurrence was not based on a thyroglobulin level or follow-up imaging, which means that the number of patients with recurrent disease is probably underestimated in both groups.

Despite these limitations, the results of this study indicate that those patients who underwent a surgeonperformed ultrasound had a statistically lower rate of recurrence at 5 years $(0$ vs. $12 \%)$ compared to patients who had a preoperative ultrasound in a radiology department. Furthermore, the mean time to recurrence was less than 1 year (11 months), suggesting that there may have been unrecognized disease present at the time of the index operation and the "recurrence" was actually persistent disease.

Depending on the sonographer, preoperative ultrasound may miss occult nodal metastases, even those in the lateral compartment. ${ }^{21}$ Theoretically, those initially occult neck nodal metastases that are not detected or removed at the index thyroid operation may become clinically relevant during the surveillance period and necessitate further surgery or other treatments. Therefore, superior preoperative detection (and extirpation) of nodal disease should lead to better locoregional control of disease as a result of the index operation, and should decrease recurrence, and possibly the need for radioiodine or reoperation. ${ }^{22}$ The findings from this study should encourage other investigators to explore the utility of surgeon-performed ultrasound at their institution to select patients for the proper index operation.

DISCLOSURE None.

\section{REFERENCES}

1. Goropoulos A, Karamoshos K, Christodoulou A, et al. Value of the cervical compartments in the surgical treatment of papillary thyroid carcinoma. World J Surg. 2004;28:1275-81.

2. Wada N, Suganuma N, Nakayama H, et al. Microscopic regional lymph node status in papillary thyroid carcinoma with and without lymphadenopathy and its relation to outcomes. Langenbecks Arch Surg. 2007;392:417-22.

3. Randolph G, Duh QY, Heller KS, et al. The prognostic significance of nodal metastases from papillary thyroid carcinoma can be stratified based on the size and number of metastatic lymph nodes, as well as the presence of extranodal extension. ATA Surgical Affairs Committee's Taskforce on Thyroid Cancer Nodal Surgery. Thyroid. 2012;22(11):1144-52

4. Kouvaraki MA, Shapiro SE, Fornage BD, et al. Role of preoperative ultrasonography in the surgical management of patients with thyroid cancer. Surgery. 2003;134:946-54.

5. O'Connell K, Yen TW, Quiroz F, Evans DB, Wang TS. The utility of routine preoperative cervical ultrasonography in patients undergoing thyroidectomy for differentiated thyroid cancer. Surgery. 2013;154(4):697-701

6. Ji YB, Lee DW, Song CM, Kim KR, Park CW, Tae K. Accuracy of intraoperative determination of central node metastasis by the surgeon in papillary thyroid carcinoma. Otolaryngol Head Neck Surg. 2014;150:542-7.

7. Wu LM, Gu HY, Qu XH, et al. The accuracy of ultrasonography in the preoperative diagnosis of cervical lymph node metastasis in patients with papillary thyroid carcinoma: a meta-analysis. Eur J Radiol. 2012;81:1798-805.

8. Shin LK, Olcott EW, Jeffrey RB, Desser TS. Sonographic evaluation of cervical lymph nodes in papillary thyroid cancer. Ultrasound Q. 2013;29:25-32.

9. Kim DW, Choo HJ, Lee YJ, Jung SJ, Eom JW, Ha TK. Sonographic features of cervical lymph nodes after thyroidectomy for papillary thyroid carcinoma. J Ultrasound Med. 2013;32: 1173-80.

10. Park JS, Son KR, Na DG, Kim E, Kim S. Performance of preoperative sonographic staging of papillary thyroid carcinoma based on the sixth edition of the AJCC/UICC TNM classification system. AJR Am J Roentgenol. 2009;192:66-72. 
11. Yoo YH, Kim JA, Son EJ, et al. Sonographic findings predictive of central lymph node metastasis in patients with papillary thyroid carcinoma: influence of associated chronic lymphocytic thyroiditis on the diagnostic performance of sonography. $J$ Ultrasound Med. 2013;32:2145-51.

12. Mazzaglia PJ. Surgeon-performed ultrasound in patients referred for thyroid disease improves patient care by minimizing performance of unnecessary procedures and optimizing surgical treatment. World J Surg. 2010;34:1164-70.

13. Solorzano CC, Carneiro DM, Ramirez M, Lee TM, Irvin GL, 3rd. Surgeon-performed ultrasound in the management of thyroid malignancy. Am Surg. 2004;70:576-80.

14. Stulak JM, Grant CS, Farley DR, et al. Value of preoperative ultrasonography in the surgical management of initial and reoperative papillary thyroid cancer. Arch Surg. 2006;141:489-94.

15. Cooper DS, Doherty GM, Haugen BR, et al. Revised American Thyroid Association management guidelines for patients with thyroid nodules and differentiated thyroid cancer. Thyroid. 2009;19:1167-214.

16. Tuttle RM, Ball DW, Byrd D, et al. Thyroid carcinoma. J Natl Compr Cancer Netw. 2010;8:1228-74.
17. Cisco RM, Shen WT, Gosnell JE. Extent of surgery for papillary thyroid cancer: preoperative imaging and role of prophylactic and therapeutic neck dissection. Curr Treat Options Oncol. 2012;13: $1-10$.

18. Lee CY, Snyder SK, Lairmore TC, Dupont SC, Jupiter DC. Utility of surgeon-performed ultrasound assessment of the lateral neck for metastatic papillary thyroid cancer. J Oncol. 2012;2012: 973124.

19. Mendez W, Rodgers SE, Lew JI, Montano R, Solorzano CC. Role of surgeon-performed ultrasound in predicting malignancy in patients with indeterminate thyroid nodules. Ann Surg Oncol. 2008;15:2487-92.

20. Rosario PW. Ultrasonography for the follow-up of patients with papillary thyroid carcinoma: how important is the operator? Thyroid. 2010;20:833-4.

21. Shim MJ, Roh JL, Gong G, et al. Preoperative detection and predictors of level $\mathrm{V}$ lymph node metastasis in patients with papillary thyroid carcinoma. Br J Surg. 2013;100:497-503.

22. Marshall CL, Lee JE, Xing Y, et al. Routine pre-operative ultrasonography for papillary thyroid cancer: effects on cervical recurrence. Surgery. 2009;146:1063-72. 